\title{
RESOLUTION OF THE PSR 1919+21 MAGNETOSPHERE EMITTING REGION
}

\author{
O. A. Kuz'Min \\ Astro Space Center, Lebedev Physical Institute, Academy of Sciences
}

\begin{abstract}
Regular structure with 10 to $50 \mathrm{kHz}$-scale was detected in the radio scintillation spectrum of PSR1919+21. This regular structure may be a simple interferometric pattern formed by refractive multi-ray propagation between the pulsar and observer. It was found that the pulsar PSR 1919+21 radio emission scintillation spectrum phase changes significantly across the average profile. Within the refractive scattering scenario this phase-longitude dependence corresponds to a transversal shift of the magnetosphere emitting region of about $6 \times 10^{8} \mathrm{~cm}$. The radio emission altitude is estimated to be near the light cylinder.
\end{abstract}

\section{Introduction}

Now a dozen pulsars are known, for which the scintillation spectra show sufficiently regular structure. On the basis of the simple form of the scintillation spectrum Ewing et al. (1970) assume that the regular spectrum is the interference pattern, formed by interference of a few rays-direct and refracted. Every pair of rays reaching the observer will form regular structure of the scintillation spectrum. The scintillation spectrum phase of this "interstellar interferometer" as well as the interference fringe phase of an interferometer must depend on the emitting source position.

In the work of Cordes, Pidwerbetsky and Lovelace (1986) it was pointed out that refraction scattering may be used to resolve spatially the pulsar magnetosphere. Later Wolszczan and Cordes (1987) reported the first successful application of this technique to the pulsar PSR 1237+25.

\section{Observations and data analysis}

Four sets of observations of PSR $1919+21$ during one month at a frequency of about $100 \mathrm{MHz}$ were used for this analysis. Observations and preliminary data processing were carried out using the predetection dispersion removal technique; the resulting signal for analysis was within a $100-\mathrm{kHz}$ band and has a temporal resolution $10 \mu \mathrm{s}$.

At the next data analysis stage the qualitative parameters of the PSR 1919+21 scintillations were determined. Scintillation dynamic spectra were computed for each observation set. The regular structure of the scintillation spectra was found in each set with typical frequency scales from 10 to $50 \mathrm{kHz}$. An example of a quasi-periodic scintillation spectrum with the period $16.7 \mathrm{kHz}$ is shown in figure 1.



Figure 1a Dynamic spectrum of PSR 1919+21. Autocorrelation function in the temporal domain.

For the second stage the dependence of the regular frequency structure phase vs. average profile 




Time Lag (seconds)

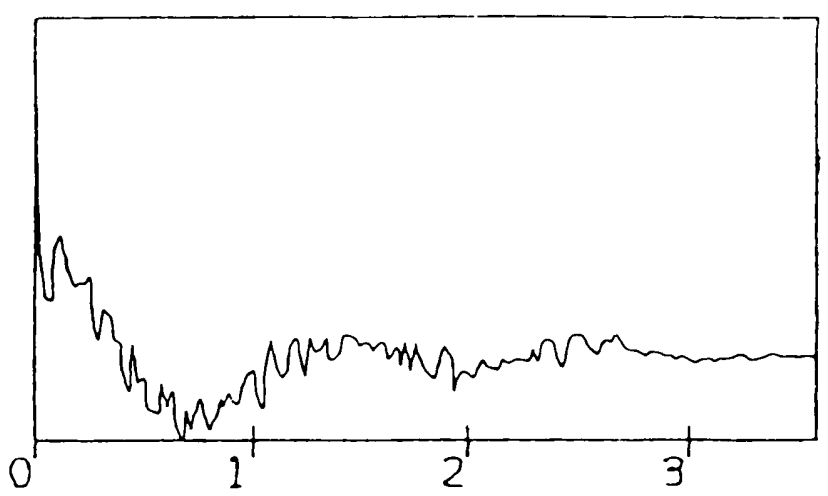

Time Lag (minutes)

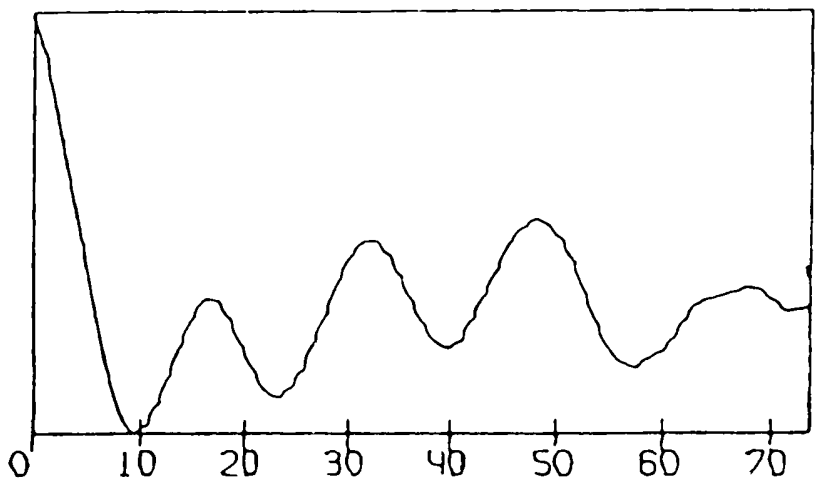

Frequency $\operatorname{Lag}(\mathrm{KHz})$

Figure 1b Dynamic spectrum of PSR 1919+21. Autocorrelation function in the spectral domain.

longitude was analyzed. Each individual impulse was divided into 10 longitudinal windows of $5.12 \mathrm{~ms}$ duration and scintillation spectra were computed in every window. After correlating the spectra of each window with the spectrum of some distinct window and averaging through all pulses of the observational set, the scintillation phase-longitude dependence was analyzed. Significant phase changes along pulse longitude were found in two observational sets and are shown in figure 2. The total



Figure 2 Upper plot: i verage profile of PSR 1919+21; middle and bottom plots: scintillation phase vs. pulse longitude for observation dates 24 February 1980 and 8 February 1980.

phase change is $0.4 \pm 0.15$ and $0.6 \pm 0.25$ radians for observational sets recorded on 8 and 24 February 1980 . Within the errors of scintillation spectra phase, the determination of phase-longitude dependence has a similar character for both data sets.

\section{Discussion}

The scintillation spectra phase dependence vs. pulse longitude found for PSR 1919+21 has the following consequences:

1. Using an interstellar interferometer with a base line of several $A U$, an angular resolution of better than $10^{-6}$ arc sec was achieved, and the magnetosphere of PSR 1919+21 was resolved.

2. The regular spectra phase changing on the pulse is due to the instantaneous emitting re- 
gion regular displacement in the pulsar magnetosphere.

What is the nature of the emitting region displacement during the pulse?

It was pointed out (Cordes, Weisberg, and Boriakoff 1983) that in the "hollow cone" model the non-radial magnetic field structure causes the instantaneous emitting region to be shifted by a distance $\Delta x$ when the pulsar turns an angle $\psi: \Delta x \cong$ $R_{\mathrm{em}} \psi \sin \beta / 3$, where $R_{\mathrm{em}}$ is the emitting region altitude and $\beta$ is the angle between the pulsar magnetic and rotation axes. As a consequence the different pulse components arrive at the observer from different magnetosphere regions and must have different fringe phases of the interstellar interferometer.

The total scintillation spectrum phase change across the PSR 1919+21 pulse obtained in our work makes it possible to estimate the transversal displacement $\Delta x$ of the instantaneous emitting region. For the observing dates 8 February 1980 and 24 February 1980 the corresponding transversal displacements are $5.2 \pm 1.2 \times 10^{8} \mathrm{~cm}$ and $6.4 \pm 2.7 \times$ $10^{8} \mathrm{~cm}$. Values obtained are less than $0.1 R_{\mathrm{LC}}$ for the pulsar PSR 1919+21. The scintillation spectrum phase vs. pulse longitude dependence shown in figure 2 agrees well with the linear relation between transversal displacement $\Delta x$ and pulse longitude $\psi$.

Now from the values of displacement $\Delta x$ and the total pulse duration $\psi=8^{\circ}$, the emission region altitude $R_{\mathrm{em}}$ can be estimated. For observing dates 8 February 1980 and 24 February 1980 the corresponding emission regions altitudes are $1.1 \pm 0.4 \times 10^{10} \mathrm{~cm}$ and $1.3 \pm 0.5 \times 10^{10} \mathrm{~cm}$. 